\title{
Comparative Study of Railway Drive Systems Using Bond-Graph
}

\author{
J. Lozano, J. Felez, J:M. Mera, J.D. Sanz \\ ETSI Engineering, Universidad Politécnica de Madrid \\ Madrid, Spain \\ e-mail: joseantonio.lozano@upm.es, jesus.felez@upm.es, josemanuel.mera@upm.es, juandedios.sanz@upm.es
}

Keywords: Simulation, Bond-Graph, transport, railway, energy efficiency.

\begin{abstract}
Since the first railways were built, they have not ceased to increase their capacity for transport, speed and energy consumption. This paper presents a comparative study of how present-day railway drive systems perform. Special emphasis is placed on consumption and energy efficiency. By using theoretical computer simulation models, simulations are conducted of the performance of present-day railway drive systems under certain specific boundary conditions. Bond-Graph Technique has been used to design the models. This technique is widely known and accepted for its suitability and capability for modeling dynamic systems in any field of science or technology, and particularly for modeling vehicular systems. Modeling is done systematically taking account of all the mechanical, dynamic, electrical, electromagnetic and regulation phenomena involved in the operation of railway drive systems.

A detailed study of the simulation results has enabled a comparative analysis to be conducted of the most significant variables. Important conclusions have been drawn from this analysis about the drive systems that have been shown to be comparatively more efficient.
\end{abstract}

\section{INTRODUCTION}

Since its beginnings, railway transport has not ceased to grow in capacity, speed and versatility. However, this growth has always gone hand-in-hand with a major growth in the energy consumption needed for its commercial and industrial operating purposes. But apart from these negative aspects, forms of transport have always played a major role in the wellbeing and economic rise of countries. Since his origins, man, for many varying reasons, has always felt a need to be on the move. A globalized world and an ever more accelerated economy make the aforementioned statement even more relevant.

For all the above reasons, scientists and experts need to continue working on the new technologies applied to means of transport to ensure such transport continues to evolve, offering more efficient and environmentally friendly systems.
The purpose of this paper is to present an initial study on the performance of the most commonly used present-day railway drive systems to make a comparative analysis of power and energy consumption and energy efficiency.

Modeling and simulation are done by applying BondGraph Technique, widely known and accepted for its suitability and capability for modeling dynamic systems in any field of science or technology, and particularly for modeling vehicular systems. This technique lets the different domains of science, mechanical physics, electricity, electronics, automatic regulation, etc, be shown at the same time in the same model. Taking the corresponding conceptual models, Bond-Graph models are very systematically generated. Likewise, using highly mechanical methods it is possible to obtain the behavior equations for the dynamic systems.

For railway energy consumption studies, a model has to be produced that takes account of all the phenomena involved in railway drive systems and those that affect longitudinal train dynamics. The different braking, retention and energy recovery systems used to regulate train speed also need to be considered.

Nowadays commercial trains are moved almost entirely by electric motors. Bond-Graph is equally used to model these engines and simultaneously incorporates electrical, magnetic and mechanical aspects.

Using the above models, the performance of different selected railway drive systems is simulated by computer under specific operating conditions. The results of these simulations enable a comparative analysis of the consumption and energy efficiency of each system to be analyzed. The conclusions and lines of research opened up as a result of this work are set out in the last section. It is important to point out that this work deals with a theoretical study that will need to be completed with subsequent work using standardized experimental tests.

\section{RAILWAY DYNAMICS MODEL}

The purpose of this model is not to study railway dynamics as there is a large bibliography on the matter, Ref.[9]. In order to be able to study energy consumption, it is sufficient to consider those phenomena that directly affect the longitudinal movement of the train in the direction of the track. 
As phenomena directly related to longitudinal movement, the following have been considered:

a) Longitudinal dynamics, governed by Newton's widely known Second Law:

$$
\Sigma \mathrm{F}=\mathrm{M} \cdot \mathrm{a}
$$

Where " $\Sigma F$ " is the algebraic sum of all the forces acting in the longitudinal direction of the train; " $\mathrm{M}$ ", is the total mass of the train, and "a", is the longitudinal acceleration.

b) Contact forces between wheels and rails. These are the traction or braking forces, " $\mathrm{F}_{\mathrm{t}}$ ", which let the train brake or accelerate. These forces are a direct consequence of the traction torque supplied either by the engines to the drive wheels or by the braking system.

c) Resistance forces opposing the train's forward motion, " $\Sigma F_{R}$ ", among which the following four types can be distinguished, depending on the cause that generates them:

c.1) Resistances to the rolling of the wheels on the rails.

c.2) Passive resistances due to the relative movement between the mechanical elements in contact with one another used to make the train operate.

c.3) Aerodynamic resistances, due to the movement of the train within the air surrounding it.

c.4) Resistances to the train's forward motion on gradients and curves.

If we incorporate the terms for the forces of traction and resistance to forward movement into the longitudinal dynamics equation given by Eq. 1, it can be written in the following form:

$$
\Sigma \mathrm{F}_{\mathrm{R}}+\mathrm{F}_{\mathrm{t}}=\mathrm{M}(\mathrm{dv} / \mathrm{dt})
$$

The phenomena associated with the appearance of these forces are well known in the field of railway dynamics. For this reason, we will now only present one of the expressions, which encompasses all the resistances previously enumerated, and which is commonly used for modern passenger trains, Ref.[9]:

$\Sigma \mathrm{F}_{\mathrm{R}}=\left(266,3+27,7 \mathrm{~V}+0,05168 \mathrm{~V}^{2}\right)+\left(\mathrm{r}_{\mathrm{g}}+500 / \mathrm{R}\right)(\mathrm{L}+\mathrm{Q})$

In which "V" is the speed of the train in $(\mathrm{Km} / \mathrm{h})$, " $\mathrm{rg}$ " is the upward gradient in $(\%)$, " $\mathrm{R}$ " is the radius of the curve in (m), "L" is the weight of the locomotives in (Tm) and "Q" is the towed weight of the wagons in (Tm).

Figure 1, shows the Bond-Graph model for longitudinal train dynamics and reflects the three phenomena that appear:

a) The longitudinal train movement, represented by Inertia port I, in ratio to the total train mass, "M".

b) The traction or braking forces that operate or brake the train, " $F_{t}$ ". Firstly, the Source of Effort, SE, supplies the traction torque, " $\tau$ ", coming from the engine or the braking torque coming from the braking system. This torque is transformed into the traction or braking force coming from the engine or the braking force coming from the " $F_{\mathrm{t}}$ ", via the Transformer Port, TF, whose ratio is the ratio of transmission between the wheels and the engine, " $i$ ". The laws governing this transformation are as follows:

$$
\begin{aligned}
& \mathrm{Ft} \cdot \mathrm{V}=\tau \cdot \omega \\
& \mathrm{Ft} \cdot \mathrm{i}=\tau \\
& \mathrm{V}=\omega \cdot \mathrm{i}
\end{aligned}
$$

The Equation, Eq.(4), is the expression of the principle of the conservation of energy, where " $\mathrm{V}$ " is the longitudinal speed of the train and " $\omega$ " is the engine speed.

c) The resistances to forward motion are represented by the Resistance port, $\mathrm{R}$. This port will have a variable ratio so that expression Eq. (8) will be fulfilled.

The three ports comprising the Bond-Graph are brought together in a type " 1 " junction, since the three phenomena are produced at the same speed, which is the train's forward speed.

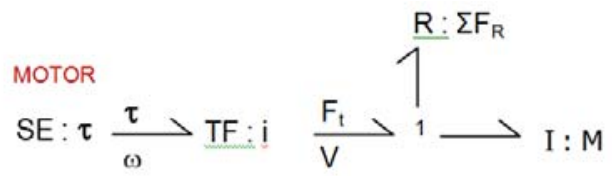

Figure 1.- Bond-Graph diagram of longitudinal train dynamics.

\section{RAIL DRIVE MOTORS}

Save for a few very specific exceptions, nowadays commercial railways are mainly moved by electric motors, Refs. [2] and [3]. The electric motors used for rail drive are basically three types:

1) In-series excitation direct current electric motors.

2) Independent excitation direct current electric motors.

3) Alternating current electric motors.

\subsection{Direct current motors}

A direct current electric motor basically consists of two components, a stator or inductor, or a rotor or armature, (see Figure 2.a). The stator's mission is to generate a magnetic field at the core of which, the rotor is inserted. This magnetic field of the stator is generated by windings through which an electric current is made to flow. An electric current is also made to flow in the rotor. As this is immersed in the magnetic field generated by the stator, the electric current conductor experiences mechanical forces that make the rotor rotate on its shaft. The difference between the different types of direct current electric motors lies in the way the stator and rotor circuits are connected; this is known as "excitation type". If the circuits are connected in series, this gives rise to the "direct current motor with in series excitation". If said circuits are connected in parallel, we get the "direct current motor with in parallel excitement". This last type is practically no longer used as it has been replaced by the "direct current 
motor with continuous excitation", where the stator and rotor winding are not interconnected and are supplied with two independent voltage sources.

Figure 2.a shows the most commonly used electromechanical layout to depict a direct current motor with independent excitement. Figure 2.b shows the equivalent Bond Graph diagram. Other already published models were taken from the references [4], [5] and [9] to construct it. These models normally convert electrical energy to mechanical energy, assuming the energy loss in the magnetic fields to be negligible. This is valid for practically all applications. This paper includes behavior of magnetic fields in order to consider the losses produced in them. This does not involve a large increase in the model's complexity but does allow a more efficient energy study to be carried out.

Described below are the general equations defining the behavior of the direct current motor with independent excitation. Also described is how to generate the Bond Graph model shown in Figure 2.b. Firstly, we shall assume that the electric motor takes the electrical energy from the overhead line or catenary. This electrical energy supply is represented using the Sources of Effort "SE", with ratio " $\mathrm{U}_{\mathrm{e}}$ " for the stator and " $\mathrm{U}_{\mathrm{r}}$ " for the rotor.

The electrical current voltage in the circuit of stator " $\mathrm{U}_{\mathrm{e}}$ " is used to overcome the ohmic resistances " $\mathrm{R}_{\mathrm{e}}$ " and to generate a magnetic field, " $\Phi$ " in the winding. The voltage supply is furnished by the Source of Effort Port, "SE" with parameter " $\mathrm{U}_{\mathrm{e}}$ ", and the ohmic resistances are represented by the Resistance port " $R$ " with parameter " $R_{e}$ ". Winding behavior is usually accomplished using an Inertia port. But in this case, in order to be able to consider the magnetic losses produced in the air-gap and the space between the stator and the rotor, the electrical energy reaching the winding is firstly converted into magnetic energy.

The equations governing the transformation of electrical energy into magnetic energy in the stator winding are:

$$
\begin{aligned}
& \mathrm{N}_{\mathrm{b}}(\mathrm{d} \Phi / \mathrm{dt})=\mathrm{U}_{\mathrm{b}} \\
& \mathrm{M}=\mathrm{N}_{\mathrm{b}} \mathrm{I}_{\mathrm{e}}
\end{aligned}
$$

Where " $\Phi$ " is the magnetic flux generated in the stator winding, " $\mathrm{U}_{\mathrm{b}}$ " is the voltage to which it is subjected and " $\mathrm{N}_{\mathrm{b}}$ " is its number of turns. " $\mathrm{M}$ " is the induced magnetomotive force and " $\mathrm{I}_{\mathrm{e}}$ " is the electric current strength in the winding.

This transfer of electrical into magnetic energy is represented in the Bond Graph using the port "GY" with ratio " $1 / \mathrm{N}_{\mathrm{b}}$ ".

In the electrical field, the stator winding is represented in the Bond Graph by a Compliance port " $\mathrm{C}$ ", in ratio to the reluctance of the magnetic field " $\mathrm{R}$ ", in such a way that the relation ship between the flux of the magnetic field and the magnetomotive force " $\mathrm{M}$ " is given by the expression:

$$
\mathrm{M}=\int \mathfrak{R}\left(\frac{\mathrm{d} \Phi}{\mathrm{dt}}\right) \mathrm{dt}
$$

Where, " $\mathrm{R}$ " is the Reluctance of the space or material through which the magnetic field crosses.

The Resistance port, $\mathrm{R}$, with ratio "P" represents the magnetic field losses produced in the air-gap of the stator and in the space between the stator and rotor.

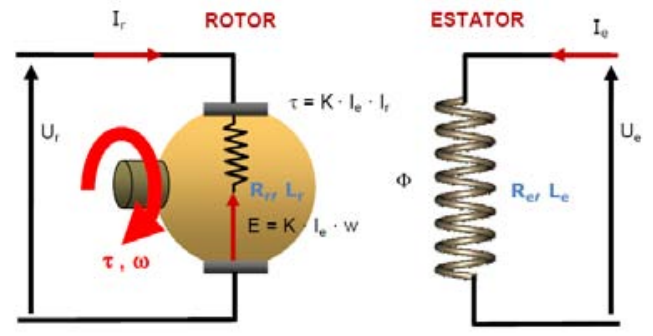

a) Electromechanical circuit diagram.

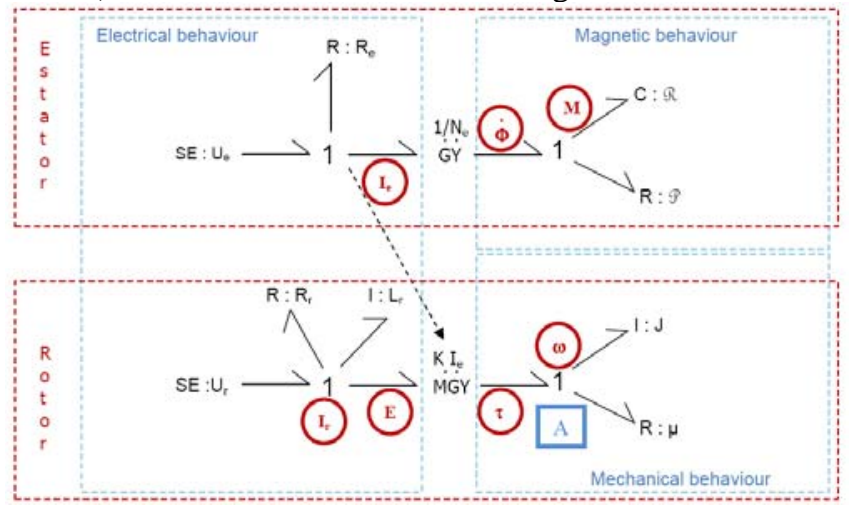

b) Bond-Graph diagram.

Figure 2. Direct current motor with independent excitation.

We will now analyze the electric circuit of the rotor or armature in Figure 2.a. The supply voltage in the " $U_{\mathrm{r}}$ " rotor circuit is supplied by a Source of Effort port "SE". In this case, the electrical energy is used to overcome the ohmic resistances represented by the Resistance port " $R: R_{r}$ ", to set up the magnetic field of the winding represented by the Inertia port "I" with inductance parameter " $\mathrm{L}_{\mathrm{r}}$ ", and to overcome the counter electromotive force " $\mathrm{E}$ " induced by the stator's magnetic field that causes the rotor to rotate. . All these elements described are subjected to the same current intensity of the rotor circuit "I,", for which reason they are connected in a " 1 "Junction.

Due to the current movement of the rotor " $\mathrm{I}_{\mathrm{r}}$ " at the core of the magnetic field generated by the stator " $\Phi$ ", mechanical forces appear that make the rotor rotate.

The equations governing the transformation of electrical into mechanical energy at the core of the rotor, Refs. [4] and [9], are:

$$
\begin{aligned}
& \tau=\mathrm{K} \cdot \mathrm{I}_{\mathrm{e}} \cdot \mathrm{I}_{\mathrm{r}} \\
& \mathrm{E}=\mathrm{K} \cdot \mathrm{I}_{\mathrm{e}} \cdot \omega
\end{aligned}
$$


Where " $K$ " is a constant, " $\tau$ " is the motor torque generated in the rotor and " $\omega$ " is its angular velocity.

These electrical into mechanical energy transformation equations are represented in the Bond Graph by the "MGY" port with variable ratio " $\mathrm{K} \mathrm{I}_{\mathrm{e}}$ ". The connection between the Bond Graph of the stator and rotor is produced through the current intensity " $\mathrm{I} e$ ". This connection is represented by a conventional broken line.

In the magnetic field, the energy is inverted to overcome the rotational inertia of the rotor, represented by the Inertia port "I", whose ratio is the moment of inertia of the rotor " $\mathrm{J}$ ". In addition, the friction losses are represented in the rotor shaft supports by the Resistance port " $\mathrm{R}$ " whose ratio is the viscous or Coulomb coefficient " $\mu$ ". In this case, these losses will be cancelled since they are already taken into account in the expression Eq. 3.

The connection of the electric motor to the longitudinal dynamic train model depicted in Figure 1 is done by eliminating the Source of Effort, SE and connecting the corresponding bond to Junction " 1 " marked by the letter "A" in Figure 2. This connection can also be seen in Figure 3.

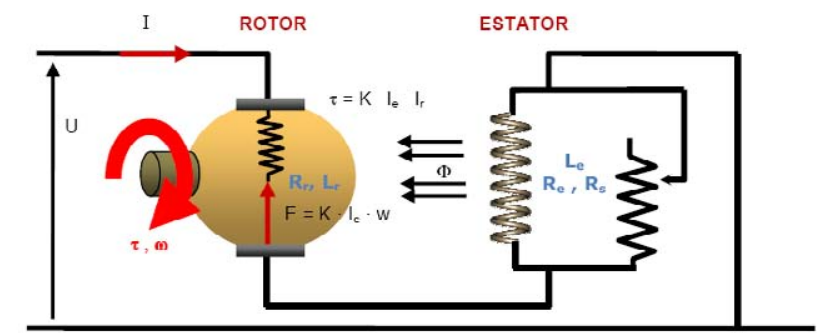

a) Electromechanical circuit diagram.

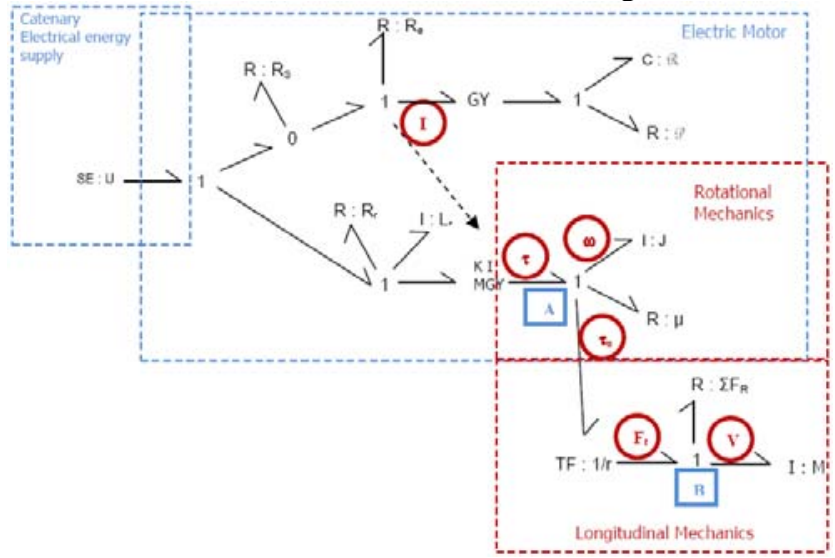

b) Bond Graph diagram.

Figure 3. DC railway drive system with in-series excitation.

Direct current motors with in- series excitation are very similar to direct current motors with independent excitation, the main difference being that the stator and rotor circuits are connected in series. Therefore, the current flowing through both circuits is the same and is also that which induces the magnetic flux that excites the rotor. As to all else, everything mentioned for the motor with independent excitation is applicable to the motor with in-series excitation.

Figure 3.a, shows the electromechanical circuit diagram for a direct current electric motor with in-series excitation, and Figure 3.b. shows the corresponding Bond Graph diagram.

\subsection{Three-phase alternating current motors}

The third type of motor used for rail traction is the asynchronous three-phase alternating current motor. This type of motor is being more and more used as it has the advantage of not having a collector, and therefore maintenance is greatly reduced.

Figure 4 Figure 6 shows the equivalent electrical circuit for each of the three-phase motor phases. The Resistance " $\mathrm{R}_{\mathrm{e}}$ " and the winding " $\mathrm{L}_{\mathrm{e}}$ " represent the behavior of the stator circuit. The Resistance " $\mathrm{R}$, " and the winding " $\mathrm{L}{ }_{\mathrm{r}}$ ", reduced to the stator circuit, represent the rotor circuit behavior. The Resistance " $R_{p}$ " and the winding " $L_{p}$ " represent the losses of hysteresis produced in the air-gap and the magnetic flux losses produced in the stator and rotor. Finally, the resistance " $R$ ' ${ }_{C}$ " is the equivalent load resistance that models the effect of the mechanical energy produced by each motor phase. The electric potential dissipated in the resistance " $R$ ' C" is equivalent to the potential generated by the electric motor in each of its phases.

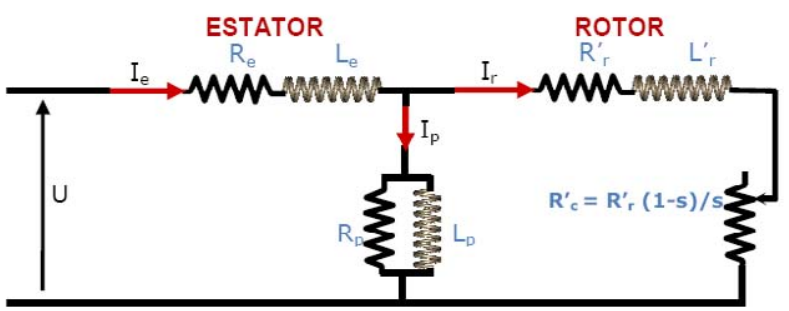

Figure 4. Equivalent circuit of an asynchronous three-phase AC motor.

Figure 5 shows the Bond-Graph model for the complete asynchronous three-phase alternating current motor. Each of the central horizontal branches in the Bond-Graph models each of the motor's phases, taking the equivalent electric circuit shown in Figure 4. The three phases are subjected to an alternating " $U$ " current, $120^{\circ}$ out of phase, using the Metatransformer ports shown to the left. The mechanical power generated by each phase is modeled by the "MGY" ports shown to the left. This rotational mechanical power is added to the " 1 " junction shown to the extreme right of the Bond Graph so it can be applied to the motor shaft modeled by the Inertia port "I" with parameter "J". The friction losses are also taken into account, and these are represented in the resistance port " $\mathrm{R}$ " with parameter " $\mu$ ". 


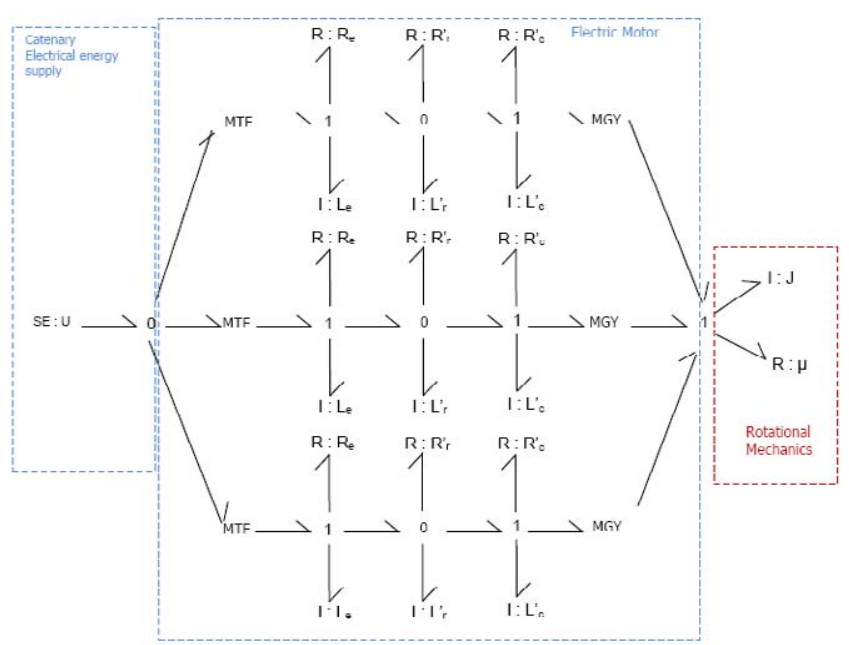

Figure 5. Bond Graph model of a three-phase AC motor.

\section{REGULATING TRAIN SPEED}

If the train is to operate properly, the first problem to be resolved is to adapt the engine torque supplied by the engine and the torque required for appropriate forward movement, to the train's speed. This is done by studying the characteristic curve of the engine and the train operation curve. These two types of curve are compared in Figure 6, where the lack of adaptation between the two curves can be seen. The train operation curve is a perfect hyperbola for high running speeds, but for low speeds it becomes horizontal since the torque cannot exceed the maximum torque due to the constraints of adherence between the wheels and the rails. This makes an additional regulation of the electric motor necessary to enable the " $\tau$ - $w$ " curve to be adapted with the greatest possible similitude to the characteristic traction curve of the train.

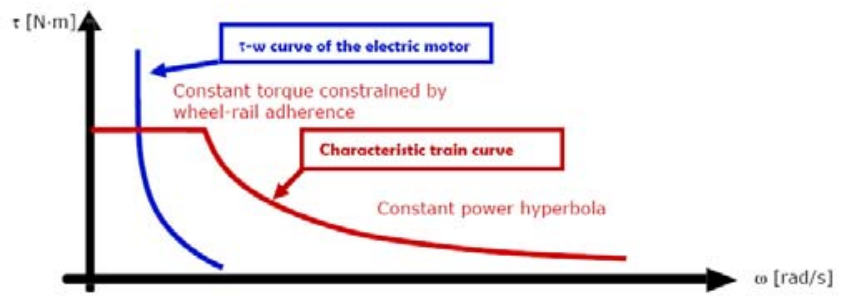

Figure 6. Electric motor $\tau-\mathrm{w}$ curve and characteristic or operation curve.

In direct current motors, in order to obtain an approximately constant motor torque in the start-up, the value of the current flowing in the rotor needs to be reduced. This is achieved by inserting resistances in series into the rotor. Once the start-up period has been completed, on attaining the constant power hyperbola, (see Figure 6), a variable resistance is mounted in series with the stator. This method is called shunting. This succeeds in reducing the intensity of the current flowing in the stator " $\mathrm{I}_{\mathrm{e}}$ ", reducing the magnetic flux " $\Phi$ ", the electromotive force induced in the rotor " $E$ " and the motor torque " $\tau$ " found on the shaft. As a result of this, the " $\tau$-w" curves of the motor, (blue in Figure 7), will move towards the right.

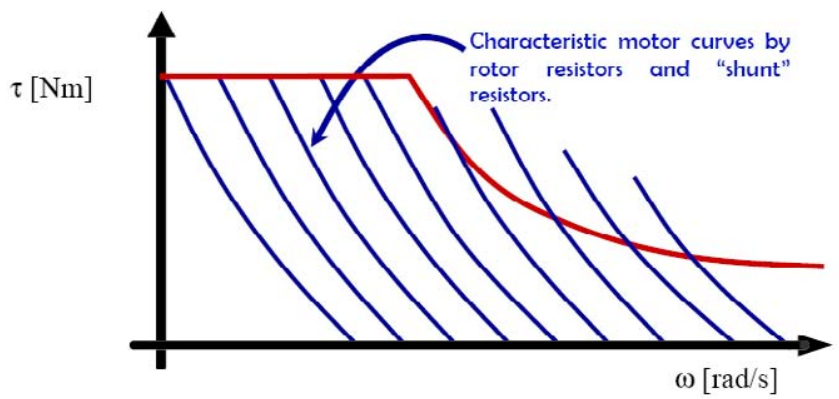

Figure 7. Characteristic curves of a direct current motor with shunting.

In the case of asynchronous alternating current motors, fitting the characteristic curve to the train operation curve is accomplished by varying the supply voltage and the current frequency by using electronic equipment known as choppers. Figure 8 shows the different motor torque curves of an asynchronous alternating current motor, found using this method and fitted to the train operation curve.

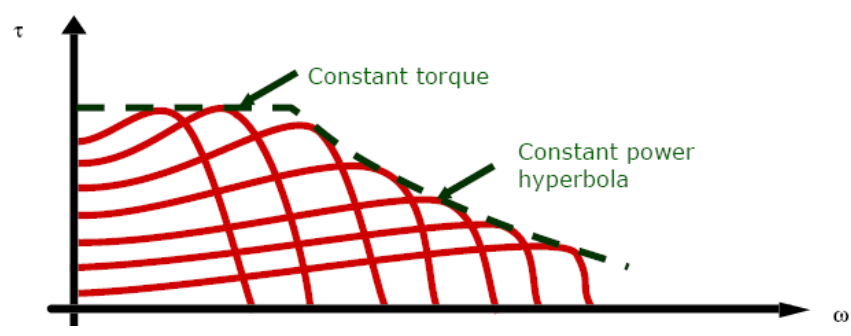

Figure 8. Characteristic curves of an alternating current motor fitted to the train operation curve.

\subsection{Braking systems}

Apart from the conventional mechanical braking system with discs or shoes, there are two braking systems that we could call electrical: rheostatic braking and regenerative braking. Mechanical braking consists in dissipating the train's mechanical energy through mechanical friction. Both types of electric brakes use the motors as generators to transform mechanical energy into electrical energy. However, there is a large difference between both. The rheostatic brake sends the current to some resistances that dissipate the electric current in the form of heat. Regenerative braking allows the electric current generated to be returned to the catenary to be used to drive other trains. So from an energy efficient point of view, the only interesting system is regenerative braking. 
However, regulating regenerative braking is not devoid of complexity. For direct current motors connected in series, (Figure 3), the current recovered is defined by the ratio:

$$
\mathrm{U}=\mathrm{E}-\mathrm{r} \cdot \mathrm{I}
$$

Or:

$$
\mathrm{I}=(\mathrm{E}-\mathrm{U}) / \mathrm{r}
$$

As the voltage drops, the current I generated increases, which causes the electromotive force $\mathrm{E}$ to increase. This makes the system unstable. To avoid this, when braking is begun, the motor is connected in independent excitation, (Figure 2). In this case a decrease in $U$ generates an increase in I, but this has no influence on E, which means operation remains stable. To perform braking while keeping the current at acceptable levels, the voltage of stator " $\mathrm{U}_{\mathrm{e}}$ " is gradually increased from the lowest level to the nominal value that corresponds to a full field.

In the case of alternating current motors used as regenerative braking, the greatest difficulty encountered is adapting the tension and frequency of the current generated to the conditions of the catenary. This is achieved with electronic systems similar to those used to regulate the operation of the motor during traction.

\section{SIMULATION RESULTS}

In order to compare the behavior of the three railway drive systems described, their performance was simulated on a real journey. The route was $68 \mathrm{~km}$ long and the profile of upward gradients and radiuses of curvature is shown in the graphs in Figure 9. Table 1, shows the specifications for the train, the locomotives and the motors. Only regenerative braking was considered in the simulations as it is obviously the most energy-efficient in every case.

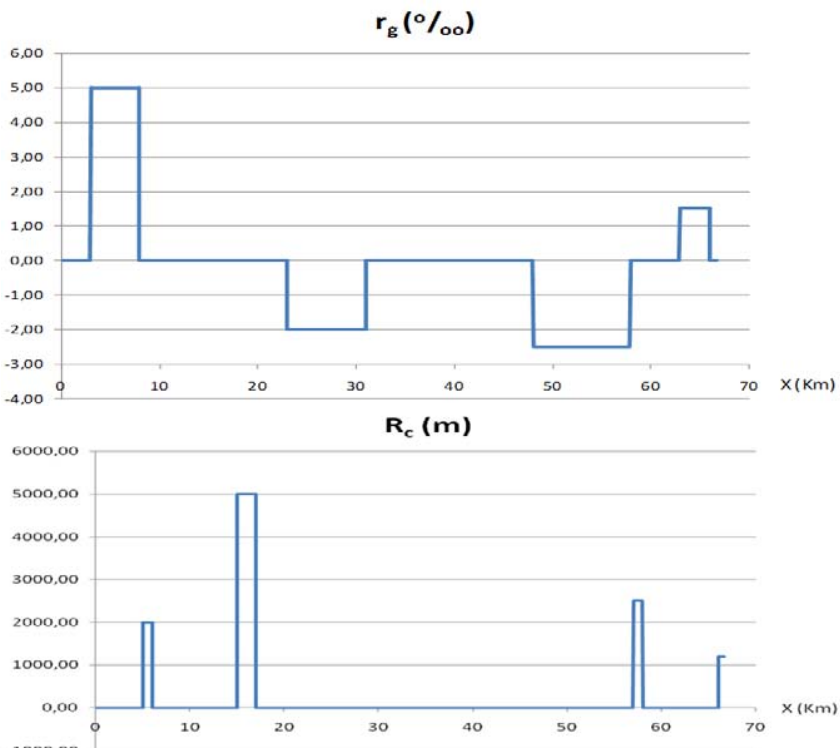

Figure 9. Upward gradient profiles " $\mathrm{r}_{\mathrm{g}}$ " $\left(\%{ }_{\mathrm{oo}}\right)$, and radiuses of curvature $R_{c}$ in $(m)$, over the route considered.
Table 1. Train specifications. Data utilized in the simulations.

\begin{tabular}{|l|}
\hline General train specifications: \\
\hline Train type: High speed / Maximum speed: $300 \mathrm{Km} / \mathrm{h}$. \\
Track width: $1437 \mathrm{~mm}+5 \mathrm{~mm} /-3 \mathrm{~mm}$ \\
Total mass: $421 \mathrm{Tm}$. / Maximum number of passengers: 337 \\
Maximum static mass per axle under normal load: $17.2 \mathrm{Tm}$. \\
Maximum dynamic load per wheel: $175 \mathrm{kN}$. \\
Train composition: 2 locomotives, 8 wagons on 9 bogies. \\
Compressed air disc brake / Electric regenerative braking. \\
\hline Locomotive specifications: \\
\hline Total length: $22.150 \mathrm{~m}$. / Total width: $2.814 \mathrm{~m}$. \\
Maximum height from rail: $4.100 \mathrm{~m}$. \\
Unit mass: 67.8 Tm. \\
Distance between bogie axles: $3.000 \mathrm{~m}$. \\
Distance between bogies: $14.000 \mathrm{~m}$. \\
Number of drive shafts (motors): 4 \\
Nominal diameter of wheels: $0.920 \mathrm{~m}$. \\
Maximum driving force per axle: $67.5 \mathrm{kN}$. \\
\hline Motor specifications: \\
\hline Nominal voltage supply: $25 \mathrm{kV}-50 \mathrm{~Hz} / 3 \mathrm{kVCC}$. \\
Nominal power: $1100 \mathrm{~kW}$ \\
Type of motor: asynchronous three-phase AC / DC. \\
Dynamic brake: regenerative, $3 / 4$ of maximum braking power. \\
\hline
\end{tabular}

As a summary of the results obtained from the simulations, shown below are some graphs of the most significant performance variables of the trains.

Figure 10 shows the curve of resistance to forward motion as a function of train speed for different values of upward gradient and radius of curvature.

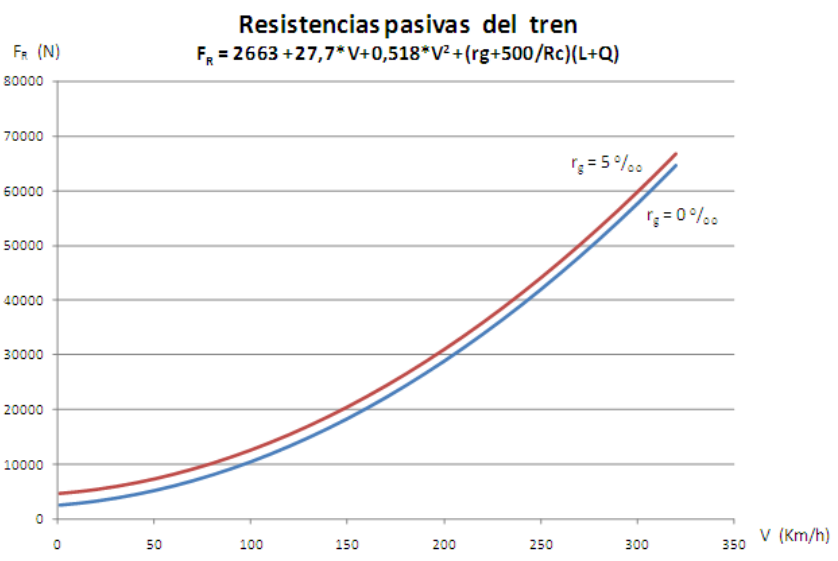

Figure 10. Resistance curve of train forward movement as a function of speed $\mathrm{V}(\mathrm{Km} / \mathrm{h})$ and of upward gradient $\mathrm{r}_{\mathrm{g}}(\%)$.

Figure 11 shows the traction force curves in accordance with train speed. To the left of the graph the saw teeth can be seen, which are due to the regulation of the motor during start-up by means of an increase in rotor resistance. Once the start-up period is completed, a drop in traction force is observed approximately in line with a constant power hyperbola. This latter is attained by shunting the stator circuit. 


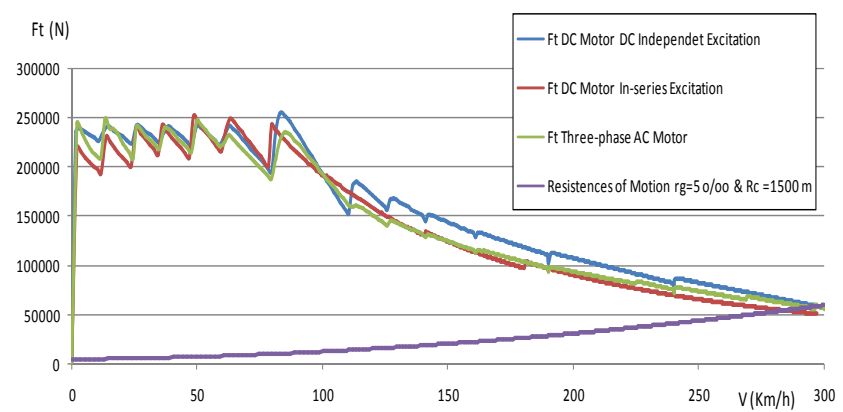

Figure 11. Total traction force curves as a function of train speed.

The tiny jumps observed in the "Ft-V" are because the regulation of motor performance was carried out at intervals according to motor rotation speed " $\omega "$. Specifically, the direct current motors were regulated by varying the shunting resistances by speed intervals. For the alternating current motors, the current frequency and shunting resistances were varied to regulate the supply voltage, taking the same speed intervals as for the direct current motors.

Figure 12, shows the curves for the electrical energy consumed over the train route. A slight drop at the end of the curves can be observed, as a result of the regenerative braking coming into operation, which transforms part of the train's mechanical energy into electrical energy and returns it to the catenary.

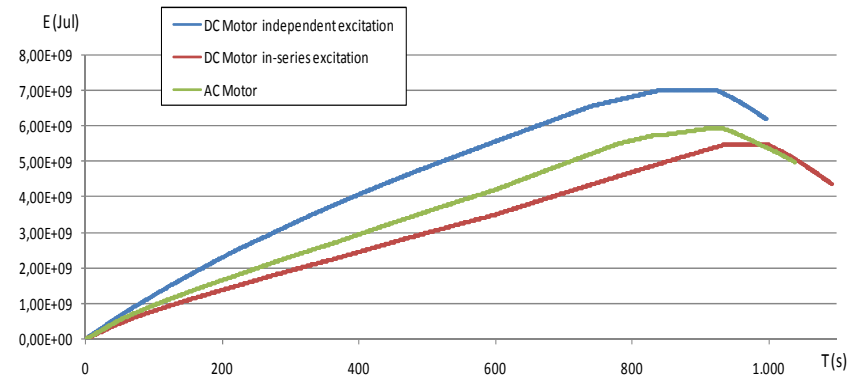

Finally, for each type of motor, the ratio of useful mechanical energy obtained in the train to the total amount of electrical energy consumed was found in order to calculate the energy performance of each type of motor, which is shown in Table 2.

Table 2. Performance of the three drive systems for the route considered.

\begin{tabular}{|l|c|}
\hline \multicolumn{1}{|c|}{ Drive system } & Performance \\
\hline Electric power motor with independent excitation & $81.8 \%$ \\
\hline Electric power motor with in-series excitation & $88.5 \%$ \\
\hline Asynchronous altemating current motor & $92.3 \%$ \\
\hline
\end{tabular}

\section{CONCLUSIONS}

The first major conclusion is that models of the three railway drive motors have been constructed - direct current motors with independent and in-series excitation, and an asynchronous alternating current motor.

In order to carry out the simulations under realistic conditions, it was necessary to model the motor regulation and train speed system and the regenerative braking system.

Bond-Graph has once again demonstrated it high potential for constructing compact models that combine the different fields of physics and technology; to be precise, in this work, electricity, magnetism and mechanics.

The behavior of the different drive systems was simulated keeping to the same criteria of regulation and considering the same standard route. However, it should be clarified that the regulation of alternating current motors has an advantage over the regulation of direct current motors. This lies in the ability to vary the electric current, which is not possible in direct current motors.

On the other hand, the regulation algorithms developed take account of a variation in shunting resistances and current frequency, as appropriate, by motor rotation speed intervals. In future works, it will be difficult for us to find algorithms that allow a continuous variation in shunting resistances and current frequency, thereby eliminating those tiny jumps detected in the "Ft-V" motor curves.

The performance of each system was calculated as the quotient of the useful mechanical energy obtained in the displacement of the train and the total electrical energy consumed. The best performance in relative terms from the simulation of the three types of motor was shown to be that of the asynchronous three-phase motor.

On the other hand, the type of motor showing the most favorable force-speed curve and allowing faster acceleration is the direct current motor with independent excitation, but its performance was worse compared to the other two types of motors.

The performances must be understood as being for a train of the characteristics considered and for the standard route adopted. In reality, trains will be able to offer even better performances depending on the route and how the motor regulation is optimized and the speed regulated on the journey. For this reason, the performances obtained should be understood in terms relative to the conditions applied in the simulations.

It should be pointed out that this first work has only attempted to conduct an initial theoretical comparative study of the performance and energy consumption of the most upto-date railway drive systems. These studies have led us to a detailed understanding of how railway drive systems perform and the problems of regulating them. Experimental information has not been dealt with, so it will be necessary to continue to carry out studies that include empirical data 
and validation tests in order to be able to confirm the results presented in this work.

\subsection{Future lines of research.}

As a result of the above conclusions, new lines of research are being opened up:

- The development of new models that take account of energy losses in more detail.

- Designing validation tests for the theoretical models developed.

- Developing more accurate regulation algorithms that will enable motor performance to be optimized and reduce energy consumption and environmental impact.

\section{References}

[1] Esperilla, J.J., Romero, G., Félez, J., Carretero, A. (2007). "Bond Graph simulation of a hybrid vehicle". Minutes of the International Congress of Bond Graph Modeling, ICBGM’07.

[2] Hata, H. (1998). "Railway Technology Today 4. What Drives Electric Multiple Units?”. Japan Railway \& Transport Review 17.

[3] Iwnicki, S. (2006). "Handbook of Railway Dynamics". Ed. Taylor \& Francis Group. London.

[4] Karnopp, D. (2005). "Understanding Induction Motor State Equations Using Bond Graphs". Minutes of the International Congress of Bond Graph Modeling, ICBGM’05.

[5] Karnopp, D.; Margolis, D.; Rosenberg, R. (2000). "System Dynamics: Modeling and Simulation of Mechatronic systems". Ed. John Wiley \& Sons, LTD $\left(2^{\mathrm{a}}\right)$. Chapter 11. ISBN: 978-0-471-33301-2.

[6] Kato, I.; Terumichi, Y.; Adachi, M.; Sogabe, K. (2005). "Dynamics of Track/Whell Systems on High-Speed Vehicles". Journal of Mechanical Science and technology, Vol 19, $\mathrm{N}^{\circ} 1$, <Special Edition> pp 328335.

[7] Pfeiffer, F.; Wriggers, P. "Lecture Notes in Applied and Computational Mechanics”. Ed. Springer. Vol. 40. 2008.

[8] Umesh, B.; Umanand, L. (2008). "Bond graph model of doubly fed three phase induction motor using the Axis Rotator element for frame transformation". Simulation Modeling Practice and Theory 16, Pag. 1704-1712.

[9] Vera, C. (2004). "Curso de Ferrocarriles". Escuela Técnica Superior de Ingenieros Industriales de Madrid. Universidad Politécnica de Madrid. 